\title{
Blob Detection Based on Soft Morphological Filter
}

\author{
Weiqing TONG ${ }^{\dagger a)}$, Haisheng $\mathrm{LI}^{\dagger \mathrm{b})}$, Nonmembers, and Guoyue $\mathrm{CHEN}^{\dagger \dagger}$, Member
}

SUMMARY Blob detection is an important part of computer vision and a special case of region detection with important applications in the image analysis. In this paper, the dilation operator in standard mathematical morphology is firstly extended to the order dilation operator of soft morphology, three soft morphological filters are designed by using the operator, and a novel blob detection algorithm called SMBD is proposed on that basis. SMBD had been proven to have better performance of anti-noise and blob shape detection than similar blob filters based on mathematical morphology like Quoit and N-Quoit in terms of theoretical and experimental aspects. Additionally, SMBD was also compared to LoG and DoH in different classes, which are the most commonly used blob detector, and SMBD also achieved significantly great results.

key words: blob detection, soft morphological filter, mathematical morphology, Quoit filter

\section{Introduction}

The blob normally refers to the region where there is color or grayscale different from surrounding domains. Since one blob represents a region, and it is featured with better stability and stronger anti-noise ability than those of simple corner point. Blob detection is an important technology in computer vision which has been applied in numerous domains including medical image processing [1]-[4], video surveillance [5], [6], robotics [7]-[9], aviation systems [10]-[12], etc.

LoG (Laplacian of Gaussian) [13], as an extremely common blob detector, is effective for the detection of radial symmetric blob, while its time complexity is relatively high. To reduce time complexity of LoG, DoG (Difference of Gaussian) blob detector was proposed [14], where the operation approximation of DoG was used to replace Gaussian Laplace operation of LoG. In order to improve the detection performance of LoG in radial asymmetric blobs, gLoG (generalized Laplacian of Gaussian) blob detector is proposed [15]. gLoG extended the detection to the rotating asymmetric structure with different Gaussian kernels, thereby detecting the blobs with general elliptical structures

\footnotetext{
Manuscript received November 12, 2018.

Manuscript revised April 14, 2019.

Manuscript publicized October 2, 2019.

${ }^{\dagger}$ The authors are with School of Computer Science and Software Engineering, East China Normal University, Shanghai,

${ }^{\dagger \dagger}$ The author is with Faculty of Electronics and Information System, Akita Prefectural University, Yurihonjo-shi, 015-0055

a)E-mail: wqtong@cs.ecnu.edu.cn

b)E-mail: hsli@cs.ecnu.edu.cn (Corresponding author) DOI: 10.1587/transinf.2018EDP7385
} China. Japan. in rotational symmetry and asymmetry. Furthermore, Miao et al. [16] proposed a blob detection algorithm based on Rank Order Laplacian of Gaussian (ROLG), guaranteeing the robustness of the filter to noise by setting the rank order sorting operation.

Another commonly used blob detector is DoH (Determinant of Hessian) [13], in which Hessian Matrix is used, where the determinant of the matrix is used to detect blobs on a single scale. In order to extract smaller scale blobs from the image, two blob detectors including HLoG (Hessianbased Laplacian of Gaussian) [17] and HDoG (Hessianbased Difference of Gaussian) [4] are proposed on the basis of DoH.

The above-mentioned blob detection algorithms are not affine invariant. Under this context, the affine co-variant blob detection algorithm was studied, among which the most influential research was the characteristic detection method of maximally stable extremum regions (MSER) proposed by Matas [18]. The watershed idea was used to detect the most stable local region of gray in the image, after which the detected region was rotated and normalized, finally acquiring local features with affine invariance in strict sense. MSER is to find the blob region meeting the measure of "the minimum area change rate" and not to find the blob shape meeting the specified size.

In addition to three classic blob detectors including LoG, DoH and MSER and their variants, there are other types of blob detection algorithms. Though the research to the blob detection has been going on, most of the research destination (especially the research in the recent years) is not to pursue how to detecting the blob shape, rather than obtaining the anchor point by detecting the blob meeting some kind of measure, and these anchor points are often used for follow-up image processing such as image registration and 3D reconstruction of sparse point cloud. Duval-Poo et al. [19] proposed a blob detector SBD (with scale invariance) and the local feature descriptor SLD based on Shearlet (Shearlet is a kind of directional multi-scale framework). After transforming the images into the Shearlet space, SBD will find the local extreme value points in the $3 \times 3 \times 3$ field of the neighboring scale space, and such extreme value points have scale invariance property. That is to say, though SBD is called the blob detector, it is not used to detect the blobs meeting specified size in the image domain, and it is used to find the local extreme value points in Shearlet space. Takacs et al. [20] proposed a kind of RIFF feature detection algorithm based on the radial gradient transformation, which has 
rotational invariance. The position of the feature points is obtained by DOB (difference of boxes), and it is to find out the local extreme value points in the $3 \times 3 \times 3$ field of the neighboring scale space. That is to say, as a kind of feature descriptor, the feature point detecting of RIFF uses DOB algorithm, and though this algorithm is called the blob detector, it is actually used to detect the extreme value points in the local area, rather than to detect the blobs meeting the specified size in the image domain.

In addition to the blob detector described above, there is a type of blob detection filter based on mathematical morphology. Quoit Filter, proposed in Ref. [1], has better shape detection capability on radial symmetric blobs. Due to high time complexity of Quoit Filter, N-Quoit Filter [2] is proposed to improve the calculation speed. But these two algorithms showed two main disadvantages: Firstly, it was weak in anti-noise ability; secondly, although it can be used for locating blobs, and it was poor in detecting the shape of the blobs.

On the basis of studies in Quoit and N-Quoit blob filter based on mathematical morphology, this paper proposes a soft morphological blob detector (SMBD) in order to improve their deficiencies. Firstly, basic operator dilation in standard mathematical morphology was extended to soft morphological order dilation, then the order dilation operator was utilized to construct two soft morphological filters including SMQ_A and SMQ_B, the former of which was used to locate the blobs, while the later one was used to detect the blob shape. Finally, a complete blob detection algorithm SMBD was designed by utilizing SMQ_A and SMQ_B filters. To verify the performance of SMBD, the experiments were conducted by comparing four methods including Quoit, N-Quoit, LoG and DoH on two blob image sets to our algorithm in this paper. The experiments showed that SMBD was obviously better both than other four methods in terms of anti-noise and blob shape detection.

The blob detection algorithm SMBD we proposed is not to detect the anchor point based on some kind of measure criterion, and it is used to detect the blob shape meeting the specified size. SMBD is a kind of improvement to the existing Ouoit and N-Quoit, and it is established on the foundation of mathematical morphological theory. Therefore, SMBD should be compared with the algorithm of the same kind. Besides, as is pointed out in the Ref. [21], LoG and $\mathrm{DoH}$ are the most widely-used algorithms in the application field of blob detecting of medical images, therefore, it is necessary to compare SMBD with these two representative algorithms although they have different principles. For the reasons above, we have selected four kinds of algorithms above, and haven't compared the other blob detecting methods proposed in the recent years.

Filter principles of Quoit and N-Quoit will be analyzed in Sect. 2, after which our blob detection method will be detailed in Sect. 3 and the results of the comparison will be given in Sect. 4, and finally, the conclusions of this paper will be drawn in Sect. 5 .

\section{Quoit Filter and N-Quoit Filter}

To detect isolated cancer shadow in the lungs, Yamamoto et al. [1] proposed a Quoit filter based on mathematical morphology as follows:

$$
\begin{aligned}
& h(x, y)=f(x, y) \oplus D_{(x, y)}-f(x, y) \oplus R_{(x, y)} \\
& g(x, y)=h(x, y) \oplus D_{(x, y)}-h(x, y) \oplus R_{(x, y)} \\
& D(u, v)= \begin{cases}0, & \text { if } u^{2}+v^{2} \leq r_{1}^{2} \\
-\infty, & \text { otherwise }\end{cases} \\
& R(u, v)= \begin{cases}0, & \text { if } r_{2} \leq u^{2}+v^{2} \leq r_{3}^{2} \\
-\infty, & \text { otherwise }\end{cases}
\end{aligned}
$$

Where, $f(x, y)$ represents the input image, $D(u, v)$ and $R(u, v)$ respectively denote disc and ring structural elements, $r_{1}$ denotes the radiuse of disc structural element, $r_{2}$ and $r_{3}$ respectively represent inner and outer radiuses of ring structural element, $h(x, y)$ represents the image after locating blobs, and $g(x, y)$ denotes the image acquired by the shape restoration of the blob via the located point.

The principle of Quoit Filter can be illustrated in Fig. 1. When the dilation processing is applied to process the isolated convex region (bright blob), the disc may only be anchored at its apex instead of falling off if the structural element is a disc (Fig. 1 (a)). If the convex region is subjected to the dilation processing with rink structural element, the circular ring may fall to the bottom when it fits into the convex region (Fig. 1 (b)). Therefore, the isolated convex region may be detected by calculating these two difference images. The region which is the convex region and fails to be harnessed into or is long convex region (Fig. 1 (c)) will not be detected due to extremely slight difference between the disc and the circular ring in the difference image. In other words, only the isolated convex region trapped by the circular ring may be detected by Quoit Filter.

The detection of isolated bright region by Quoit Filter is composed of two morphological transformations, where, the first morphological transformation anchors the bright blob, and the second morphological transformation restores the shape of the blob. When the bright blob takes on a hemispherical shape, the restored shape is also hemispherical, and the shape of the blob is not ideally restored if the blob is not hemispherical. Quoit Filter mainly suffers from three defects: (1) Relatively poor anti-noise ability; (2) Relatively poor blob shape recovery; (3) Relatively high time complexity.

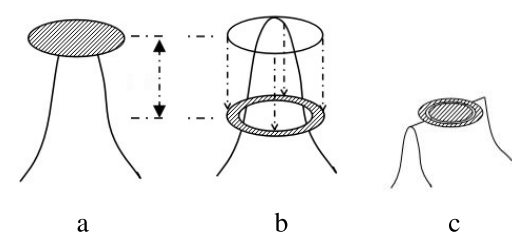

Fig. 1 Schematic diagram for Quoit filter. 
In order to improve the processing speed of Quoit Filter, Miwa et al. [2] proposed the N-Quoit Filter. It is assumed that the bright blob is featured with rotational symmetry and highly monotonic decreasing, the dilation filtering of single-point structural element may replace the dilation filtering of disc structural element, consequently greatly shortening the processing duration. The definition of $\mathrm{N}$ Quoit Filter is shown as follows:

$$
h(x, y)=f(x, y)-f(x, y) \oplus R_{(x, y)}
$$

Where $f(x, y)$ denotes the input image, $R$ represents ring structural element, and $h(x, y)$ denotes the resulting anchor image of the blob. N-Quoit is only better than Quoit in terms of the filtering speed, and it has the disadvantage of failure to overcome its noise sensitiveness like Quoit. Additionally, $\mathrm{N}$-Quoit Filter is only able to anchor the position of the blob and is incapable of detecting the shape of the blob.

For particular blob with rotational symmetry and highly monotonically decreasing, the method for automatically determining the filter size by conducting the binary distance transform (BDT) on the input image is given in Ref. [2]. Furthermore, the gray weight distance transform (GWDT) is proposed in Ref. [2] to act a method of preprocessing with the aim of improving the processing performance of N-Quoit filter on the overlapping region between isolated shadow and linear shadow (e.g the cancer shadow near blood vessel or cancer shadow traversed by blood vessel). As the preprocessing of abovementioned two distance transforms is included in the processing of special image, generally they are not considered to be a part of N-Quoit filter.

\section{SMBD}

\subsection{Order Dilation Operator}

Koskinen et al. [22] extended the dilation operator of standard mathematical morphology to soft dilation, which is mainly characterized with less sensitive to additive noise and the change in the shape requiring filtering. The definition of soft dilation is shown as follows:

$$
\begin{aligned}
& f \oplus[B, A, K](x, y)=k^{\text {th }} \text { largest value of multiset } \\
& \left\{k * f(a): a \in A_{(x, y)}\right\} \cup\left\{f(b): b \in(B-A)_{(x, y)}\right\} .
\end{aligned}
$$

The above operator in soft morphology is defined on the basis of sorting statistics, the structural element of standard mathematical morphology is replaced with the structural system $[B, A, k]$. The structural system consists of three parameters: For the sets of finite planes $A$ and $B$, $A \subset B$ is satisfied, a natural number $k$ satisfies $1 \leq k \leq$ $\min \left\{\frac{|B|}{2},|B-A|\right\}$, and $|\cdot|$ represents total number of elements in the sets. $B$ denotes the structural set, $A$ represents the hard center of $B, B-A$ generates corresponding soft contour, and $k$ represents the order index specified. The symbol $*$ indicates repeated operation, and is defined to be $k * x=$ $\overbrace{x, x, \cdots, x}^{\text {ktimes }}$, for example $\{2 * 1,2,3 * 3\}=\{1,1,2,3,3,3\}$. Obviously, when $A=\phi$ and $k=1$ are satisfied, the soft dilation degenerates into a standard dilation.

In order to design Quoit Filter in soft morphology, the order dilation operator based on the soft dilation operator is defined firstly.

Definition of order dilation:

$$
\begin{aligned}
& f \oplus[B, k](x, y)= \\
& k^{\text {th }} \text { largest value of multiset }\left\{f(b): b \in B_{(x, y)}\right\}
\end{aligned}
$$

Where, $B$ denotes a structural element, $\mathrm{k}$ represents the natural numbers satisfying $1 \leq k \leq \frac{|B|}{2}$, and the order indexes are sorted from the maximum to the minimum. Equation (7) actually turns the hard center $A$ in the structural system $[B, A, k]$ in Eq. (6) into the empty set, thereby causing the structural system to degenerate into $[B, k]$. It means that image $f$ is subjected to morphological dilation by use of soft contour $B-A$ and hard center $A$ converts to the morphological dilation of image $f$ merely by Soft Contour of structural element $B$.

\subsection{Soft Morphological Quoit Filters}

By utilizing the order dilation operator, three Quoit filters in soft morphology is firstly defined as follows.

(1) SMQ Filter:

$$
\begin{aligned}
& S M Q\left(D, R, k_{D}, k_{R}, f, x, y\right)= \\
& f \oplus\left[D, k_{D}\right](x, y)-f \oplus\left[R, k_{R}\right](x, y)
\end{aligned}
$$

(2) SMQ_A Filter:

$$
\begin{aligned}
& S M Q A A\left(D, R, k_{a}, k_{b}, k_{c}, k_{d}, f, x, y\right)=\frac{1}{k_{b}-k_{a}+1} \\
& \sum_{i=k_{a}}^{k_{b}} f \oplus[D, i](x, y)-\frac{1}{k_{c}-k_{d}+1} \sum_{j=k_{c}}^{k_{d}} f \oplus[R, j](x, y) \\
& \text { where }, k_{a}, k_{b}=1,2, \ldots,|D| / 2 ; \\
& k_{c}, k_{d}=1,2, \ldots,|R| / 2 ; k_{a} \leq k_{b}, k_{c} \leq k_{d} .
\end{aligned}
$$

(3) SMQ_B Filter:

$$
\begin{aligned}
& S M Q \_B\left(D, R, k_{m}, k_{n}, f, x, y, u, v\right)= \\
& f(u, v)-\frac{1}{k_{m}-k_{n}+1} \sum_{i=k_{m}}^{k_{n}} f \oplus[R, i](x, y)
\end{aligned}
$$

Where, $f$ denotes the image of filtered object, $k_{m}, k_{n}=$ $1, \ldots,|R| / 2, k_{m} \leq k_{n}, R$ denotes ring structural element, $D$ denotes disc structural element, and $(u, v) \in D_{(x, y)}$ (where $D$ $\notin R$ is satisfied, and $D$ and $R$ are homocentric). The working principle of the above three Quoit filters in soft morphology will be described as below.

Working principle of SMQ Filter: Intuitively, Quoit Filter actually moves concentric disc and ring to Object 
Point $P$, and then finds maximum value $V_{D}$ of the disc domain and maximum value $V_{R}$ of circular ring domain respectively. The Object Point $P$ is determined to be included in the blob region or not via the value of $V_{p}=V_{D}-V_{R}$. SMQ Filter is similar to Quoit Filter except that it finds its own specified order index in the disc and ring domain respectively rather than maximum value, consequently avoiding the effects of changes in the noise and shape effectively. SMQ is degenerated into Quoit Filter when $k_{D}$ and $k_{R}$ of SMQ Filter are set to be 1, and it degenerates into N-Quoit Filter if $D$ is set to one element. The robustness of SMQ to noise and morphological change may be adjusted by adjusting the order indexes including $k_{m}$ and $k_{n}$. SMQ Filter is mainly used for locating blob.

Working principle of SMQ_A Filter: According to the introduction to the principle of SMQ above, it is concluded SMQ is a morphological filter, in which the order index is specified, and SMQ_A denotes an extension of SMQ. When SMQ_A morphologically filters the blob with a disc and a ring structural element, it is based on the mean value of gray values corresponding to certain segment of order indexes rather the gray value corresponding to a certain order index. Therefore, theoretically SMQ_A exclusively delivers better shape robustness than SMQ based on a certain order index. When the order indexes including $k_{a}=k_{b}$ and $k_{b}=k_{d}$ are satisfied, SMQ_A degenerates into SMQ. SMQ_A Filter is mainly used for locating blob.

Working principle of SMQ_B Filter: Both SMQ and SMQ_A are exclusively used to locate the blob rather than detecting actual shape of the blob due to that the only thing to be considered is morphological change in the point rather than morphological changes in other points in the disc domain when the object point is subjected to morphological filtering. To solve this problem, SMQ_A is modified, and formerly Structural Element $D$ of the disc is decomposed into the structural element with only a single element (the dilation processing based on structural element of single elements is the image itself), and SMQ_A is applied to make morphological filtering on every point $(u, v)$ in the disc domain $(R$ is always kept to exclusively filter Object Point $(x, y)$ in such a structural element). In other words, when SMQ_B Filter filters Object Point $(x, y)$, the difference between gray value of point $(u, v)$ and response value (the right part of Eq. (10)) of point $(x, y)$ based on soft morphological filtering of $R$ is calculated within the range where the point $(x, y)$ is the center of the circle and is covered by $D$, consequently SMQ_B may detect the shape of the region of Object Point $(x, y)$ covered by $\mathrm{D}$ when the object point $(x, y)$ is morphologically filtered. The point $(x, y)$ is subjected to the processing of soft morphological filtering (right part of Eq. (10)) by structural element $R$ of circular ring within the range of the order indexes $k_{m} \sim k_{n}$, essentially, the filtering effects on the shape of the blob are changed by adjusting the response value of the filter at the point. Since the mean between order indexes $k_{m} \sim k_{n}$ acts as response value of morphological filtering, theoretically, it is stable compared to morphological filtering based on single order index.

\subsection{The Blob Detection Algorithm}

SMQ is a Quoit Filter designed based on order dilation operator in this paper, and it involves Quoit Filter and NQuoit Filter, while SMQ_A and SMQ_B are extensions to SMQ. The bright blob detection algorithm SMBD based on SMQ_A and SMQ_B filters is shown below. To detect dark blobs, it will work to reverse the gray scale of the input image.

\section{Algorithm 1 SMBD Algorithm \\ Step 1: Parameter settings of filter \\ (1) Set the parameters $D_{A}, R, k_{a}, k_{b}, k_{c}, k_{d}$ of SMQ_A Fil- ter, where $D_{A}$ denotes disc structural element of SMQ_A Filter.}

(2) Set the parameters of SMQ_B Filter $D_{B}, R, k_{m}$ and $k_{n}$, where $D_{B}$ denotes the disc structural element of SMQ_B Filter.

(3) Set the threshold of locating blob to be $T h_{A 1}$ and the threshold of blob region to be $T h_{A 2}$, these two thresholds are mainly used to control the number of blob candidates.

Step2: SMQ_A Filtering

The input image $I(x, y)$ is subjected to the blob filtering by using the SMQ_A Filter, acquiring the image $L(x, y)$ of location point candidates:

$$
L(x, y)=S M Q A\left(D_{A}, R, k_{a}, k_{b}, k_{c}, k_{d}, I, x, y\right) .
$$

Step3: Generating the mask image $M(x, y)$

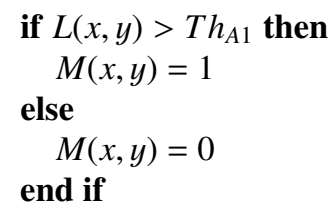

\section{Step4: SMQ_B Filtering}

Set $I_{2}(x, y)$ to be the image filtered with SMQ_B. Firstly initialize $I_{2}(x, y)$ with 0 , then traverse each effective pixel $(x, y)$ in image $I(x, y)$, and perform the following filtering processing steps with SMQ_B. Where, $v(u, v)$ denotes the filtered value of SMQ_B under the precondition of $(u, v) \in D_{B(x, y)}$.

$$
\begin{aligned}
& \text { if } M(x, y)=1 \text { then } \\
& v(u, v)=\mathrm{SMQ} \mathrm{B}\left(D_{B}, R, k_{m}, k_{n}, I, x, y, u, v\right) \\
& \text { if } v(u, v)>I_{2} \text { then } \\
& \quad I_{2}(u, v)=v(u, v) \\
& \text { end if } \\
& \text { end if }
\end{aligned}
$$

Step5: Extracting the blob shape

$$
\begin{aligned}
\text { if } I_{2}(u, v) & \geq T h_{A 2} \text { then } \\
I_{3}(u, v) & =I(u, v) \\
\text { else } & \\
I_{3}(u, v) & =0
\end{aligned}
$$




\section{end if}

Where $I_{3}(x, y)$ denotes the blob image detected.

SMBD Algorithm is divided into five steps: (1) Set all the parameters in the algorithm; (2) Conduct the locating processing on the blob in the input image which is smaller than inner radius of specified circular ring with SMQ_A Filter, and smaller radius of the disc may be valued to improve the processing speed. As the calculation quantity will be extremely great if the blob filtering is directly performed by SMQ_B, consequently SMQ_A is firstly utilized to roughly determine the center position of the blob in the algorithm; (3) Filter the candidate for the blob location point to generate the mask image; (4) Take the point valued in 1 as the center of SMQ_B Filter in Mask Image A, and then have Image I subjected to the filtering processing of SMQ_B to acquire the filtered response value image of SMQ_B, namely the response value of detected blob shape; (5) Extract the shape of the blob with the response value greater than the threshold $T h_{A 2}$ from Image I according to $I_{2}$, the filtered image of SMQ_B.

\subsection{Estimation of SMBD Parameters}

SMBD has 12 parameters (please refer to Sect. 4 for naming symbols and descriptions of SMBD parameters), and we give the general method of estimating these parameters first, then we will examine the effect of the variance of such parameters to detecting blob.

The disc structure element of SMQ_A are used to locate the center of the target blob; in general, larger $r_{A 1}$ obviously will reduce the confidence of the anchor point of the false target blob (the filtering value of SMQ_A), however, it will also increase the processing time of SMQ_A; according to the experiences, it is proper to make it $20 \% \sim 50 \%$ of the radius of target blob $r_{T} . r_{A 2}$ is the inner radius of the ring structure element, and only it is larger than $r_{A 1}$; however, on considering of proper tolerance, generally $r_{A 2}=r_{T}+2 . r_{A 3}$ is the outer radius of the ring structure element, and generally it should warrant that the circle has the width of 2 pixels, i.e., $r_{A 3}=r_{A 2}+1$. $r_{A 4}$ is the radius of the disc structure element of SMQB, and it is used to detect the shape of the target blob, therefore, $r_{A 4}$ must equal to $r_{T}$.

$k_{a}$ is the order index of the lower limit of the disc structure element of SMQ_A during the soft dilation, when SMQ_A is searching in the background area, it is mainly used to tell SMQ_A filter: the pixels of which are smaller than the order index $k_{a}$ in the area covered by SMQ_A ring might be noise. Therefore, $k_{a}$ can be set as: $k_{a}=$ (the disc area of SMQ_A) $\times$ (the average noise occupation ratio in the unit area). $k_{b}$ is the order index of the upper limit of the disc structure element of SMQ_A during the soft dilation, when SMQ_A is searching in the blob area, it is mainly used to tell SMQ_A filter: the pixels of which are greater than the order index $k_{b}$ in the area covered by SMQ_A ring might be noise. Therefore, $k_{b}$ can be set as: $k_{b}=$ (the disc area of SMQ_A $) \times(1-$ the average noise occupation ratio in the unit area). $k_{c}$ is the order index of the lower limit of the ring structure element of SMQ_A during the soft dilation, when SMQ_A is located in the background area, it is mainly used to tell SMQ_A filter: the pixels of which are smaller the order index $k_{c}$ in the area covered by SMQ_A ring might be noise. Therefore, $k_{c}$ can be set as: $k_{c}=$ (the ring area of SMQ_A) $\times$ (the average noise occupation ratio in the unit area). $k_{d}$ is the order index of the upper limit of the ring structure element of SMQ_A during the soft dilation, when the ring center of SMQ_A coincides with the blob center, it is used to tell SMQ_A: in the area covered by the ring, the pixels with the order index larger than $k_{d}$ can most represent the background property surrounding the blob area, and normally $k_{d}=3 k_{c} . k_{m}$ and $k_{n}$ are the order index of the lower limit and upper limit of the ring structural element of SMQ_B respectively, and their effects are the same as those of $k_{c}$ and $k_{d}$, therefore $k_{m}=k_{c}, k_{n}=k_{d}$.

$T h_{A 1}$ is the threshold of the anchor point of the blob, and it is used to select the correct candidate positioning for blob; it can be determined according to the average gray scale contrast in the background area in the central area and border of the blob. $T h_{A 2}$ is the threshold for extracting the blob shape and it is used to select the correct candidate blob, and it can be determined according to the average grey scale contrast of the blob shape and its surrounding background.

The content above states the general method of estimating SMBD parameters theoretically according to the working principle of SMBD, however, it is not the optimum parameter selection; how to automatically and effectively estimate SMBD parameters is the theme to be researched in the future. The estimation method on SMBD parameters above has been generalized in Table 1. From Table 1 we can see

Table 1 Estimation of SMBD parameters

\begin{tabular}{l|l}
\hline Parameter & Setting value \\
\hline$r_{A 1}$ & $r_{T} \times(20 \% \sim 50 \%)$ \\
\hline$r_{A 2}$ & $r_{T}+2$ \\
\hline$r_{A 3}$ & $r_{A 2}+1$ \\
\hline$r_{A 4}$ & $r_{T}$ \\
\hline$k_{a}$ & $\begin{array}{l}\text { (the disc area of SMQ_A) } \times \text { (the average noise occupation } \\
\text { ratio in the unit area) }\end{array}$ \\
\hline$k_{b}$ & $\begin{array}{l}\text { (the disc area of SMQ_A }) \times(1 \text { - the average noise occupa- } \\
\text { tion ratio in the unit area) }\end{array}$ \\
\hline$k_{c}$ & $\begin{array}{l}\text { (the disc area of SMQ_A) } \times \text { (the average noise occupation } \\
\text { ratio in the unit area) }\end{array}$ \\
\hline$k_{d}$ & $3 k_{c}$ \\
\hline$k_{m}$ & $k_{c}$ \\
\hline$k_{n}$ & $k_{d}$ \\
\hline$T h_{A 1}$ & $\begin{array}{l}\text { It can be determined according to the average gray scale } \\
\text { contrast in the background area in the central area and bor- } \\
\text { der of the blob. }\end{array}$ \\
\hline$T h_{A 2}$ & $\begin{array}{l}\text { It can be determined according to the average grey scale } \\
\text { contrast of the blob shape and its surrounding background. }\end{array}$ \\
\hline
\end{tabular}




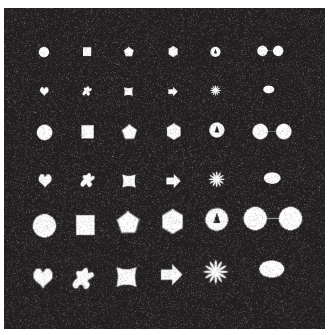

a

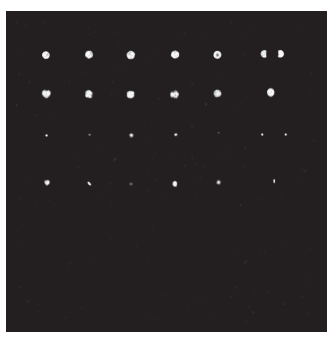

b

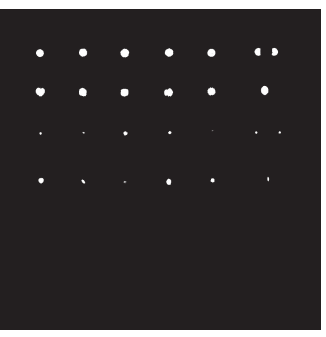

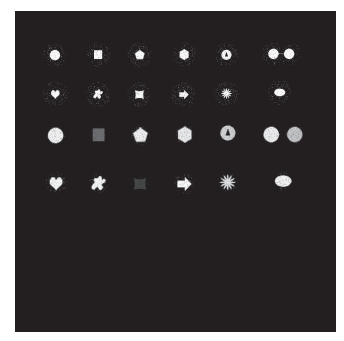

d

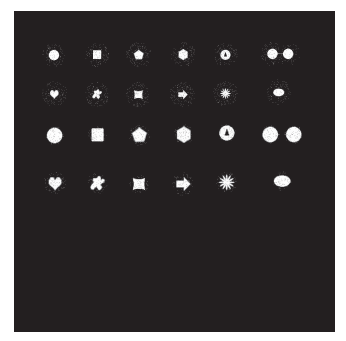

$\mathrm{e}$

Fig. 2 The images on the various intermediate processing procedure of blob detecting with SMBD. (a) is the original image $A_{2}$. (b) is the candidate anchor point of blob obtained after the filtering of SMQ_A. (c) is the image after (b) is processed with binaryzation with the threshold of $T h_{A 1}=60$. (d) is the image of SMQ_B after the blob is processed with morphology filtering at the candidate anchor points. (e) is the real blob shape extracted from the original image of which the morphology filtering value of the blob is larger than threshold $T h_{A 2}=10$.

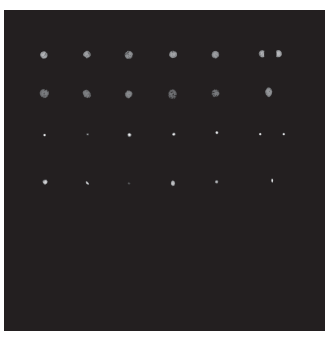

a

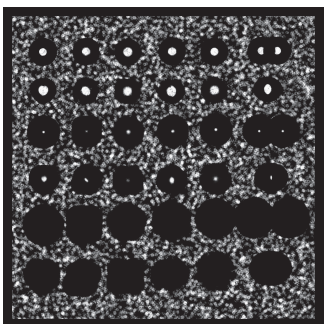

b

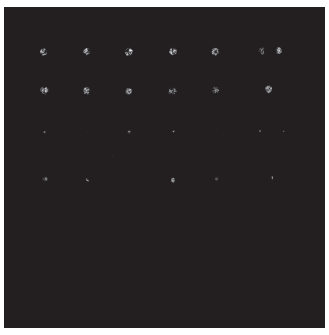

$\mathrm{c}$

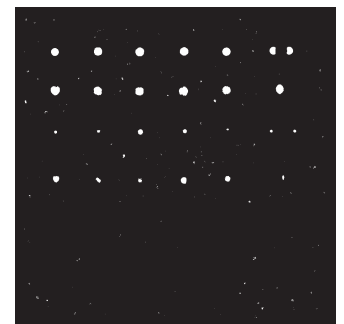

d

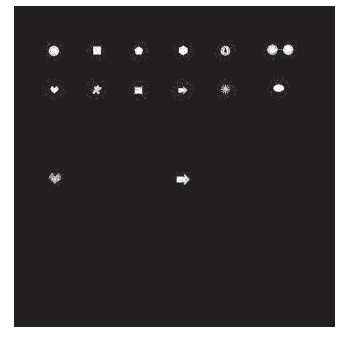

e

Fig. 3 The effect of the parameter variation of SMBD on the blob detecting. (a) is the image of the candidate anchor point of blob detected by SMBD at $r_{A 1}=20$. (b) is the image of the candidate anchor points of the blobs detected by SMBD at $k_{a}=1$ and $k_{b}=5$. (c) is the image of the candidate anchor points of the blobs detected by SMBD at $k_{c}=7$ and $k_{d}=10$. (d) is the image of the candidate anchor points of the blobs detected by SMBD at $T h_{A 1}=10$. (e) is the image of the blob shape detected by SMBD at $T h_{A 2}=250$.

that, if the size of the target blob is known, we can estimate four parameters of $r_{A 1}-r_{A 4}$; if the noise distribution of the image is known, we can estimate six parameters of $k_{a}-k_{n}$; if we know the contrast between the blob center and blob shape and the surrounding background at the blob border, we can estimate $T h_{A 1}$ and $T h_{A 2}$.

According to the method estimating SMBD parameters introduced above, we can observe the intermediate procedure of SMBD during the blob detecting. We select image $A_{2}$ in Image Set A (please refer to the instruction on Image Set $\mathrm{A}$ in 4.1), and it is the image after being applied $5 \%$ salt-and-pepper noise on image $A_{1}$ (Fig. 2 (a)). Our purpose is to detect the blobs in row 3-4 of image $A_{2}$ with the radius of 26 pixels, and the following parameters will be given according to the method of parameter estimation in the Table 1: $r_{A 1}=5, r_{A 2}=28, r_{A 3}=29, r_{A 4}=26$, $k_{a}=\left\lceil\pi \times r_{A 1}^{2} \times 5 \%\right\rceil=4, k_{b}=\left\lceil\pi \times r_{A 1}^{2} \times(1-5 \%)\right\rceil=75$, $k_{c}=\left\lceil\pi \times\left(r_{A 3}^{2}-r_{A 2}^{2}\right) \times 5 \%\right\rceil=9, k_{d}=3 k_{c}=27, k_{m}=k_{c}=9$, $k_{n}=k_{d}=27, T h_{A 1}=60, T h_{A 2}=10$.

Figure 2 (b) is the candidate anchor point of blob obtained after the filtering of SMQ_A, from the image we can see that the larger the blob area is, the smaller the corresponding area of anchor point will be. Figure 2 (c) is the image after Fig. 2 (b) is processed with binaryzation with the threshold of $T h_{A 1}$ and such processing can eliminate the wrong candidate anchor points with the filtering value less than $T h_{A 1}$. Figure $2(\mathrm{~d})$ is the image of SMQ_B after the blob is processed with morphology filtering at the candidate anchor points. Figure 2 (e) is the real blob shape extracted from the original image of which the morphology filtering value of the blob is larger than threshold $T h_{A 2}$.

Finally, we will examine the effect to the blob detecting when the SMBD parameters are improperly set. All the examination testing are based on the image $A_{2}$ and its parameters above; when a certain parameter is changed, the other parameters dont change.

Figure 3 (a) is the image of the candidate anchor point of blob detected by SMBD at $r_{A 1}=20$. Compared with Fig. 2 (b), the both are basically the same except for that the filtering value changes. It indicates that we can select $r_{A 1}$, which is far less than the radius of the target blob to obtain the candidate anchor point, and the processing speed of SMQ_A can be increased a lot. Figure 3 (b) is the image of the candidate anchor points of the blobs detected by SMBD at $k_{a}=1$ and $k_{b}=5$. Because the parameters $k_{a}$ and $k_{b}$ are not proper, a lot of noise points are regarded as the candidate anchor points of blobs. The parameters $k_{a}$ and $k_{b}$ have large effect to the correct detection of the candidate anchor points of the blobs. Figure 3 (c) is the image of the candidate anchor points of the blobs detected by SMBD at $k_{c}=3$ and 
$k_{d}=10$. Because the parameters $k_{c}$ and $k_{d}$ are not proper, some blobs havent been positioned, and the filtering value of the candidate anchor point is obviously smaller. The parameters $k_{c}$ and $k_{d}$ have large effect to the correct detecting of the candidate anchor points of the blobs. Figure 3 (d) is the image of the candidate anchor points of the blobs detected by SMBD at $T h_{A 1}=10$. Because $T h_{A 1}$ is smaller, some non-candidate anchor points of blob have also been included. Figure $3(\mathrm{e})$ is the image of the blob shape detected by SMBD at $T h_{A 2}=250$. Because $T h_{A 2}$ is larger, some correct blobs have been wrong eliminated.

\section{Comparision Experiments}

To test the performance of SMBD, two experimental image sets (noisy image set and noiseless image set) are respectively designed, and two algorithms with the same types (Quoit and N-Quoit) and two common algorithms with different types (LoG, DoH) are respectively subjected to comparison experiments.

For the convenience of documenting, named symbols for the parameters used in five algorithms are respectively given. 12 parameters of SMBD: $r_{A 1}$ and $r_{A 4}$ respectively denote the radiuses of disc structural elements of SMQ_A and SMQ_B; $r_{A 2}$ and $r_{A 3}$ respectively represent inner and outer radiuses of ring structural element; $k_{a}$ and $k_{b}$ respectively denote the order index of the lower and upper limit of the disc structure element of SMQ_A during the soft dilation; $k_{c}$ and $k_{d}$ respectively denote the order index of the lower and upper limit of the ring structure element of SMQ_A during the soft dilation; $k_{m}$ and $k_{n}$ respectively denote the order index of the lower and upper limit of the disc structure element of SMQ B during the soft dilation; $T h_{A 1}$ denote the threshold of the anchor point of the blob; $T h_{A 2}$ The threshold for extracting the blob shape. 4 parameters of Quoit: $r_{B 1}, r_{B 2}$ and $r_{B 3}$ respectively denote the radius of disc structural element, inner and outer radiuses of ring structural element, $T h_{B 1}$ represents the threshold of the response of Quoit filtering, being equivalent to $T h_{A 1}$ of SMBD. 3 parameters of N-Quoit: $r_{C 1}$ and $r_{C 2}$ denote inner and outer radiuses of ring structural element, and $T h_{C 1}$ represents the threshold of the response of N-Quoit Filter, being equivalent to $T h_{A 1}$ of SMBD. 3 parameters of LoG: $r_{D 1}, r_{D 2}$ and $r_{D 3}$ satisfy $r_{D 1}<r_{D 2}<r_{D 3}$. Where, $r_{D 2}$ denotes the radius of the target blob to be detected with LoG, and $r_{D 1}$ and $r_{D 3}$ represent radius ranges of the target blob for which LoG Filter is required to be searched. 3 parameters of DoH: $r_{E 1}, r_{E 2}$ and $r_{E 3}$ satisfy $r_{E 1}<r_{E 2}<r_{E 3}$. Where, $r_{E 2}$ denotes the radius of the target blob to be detected with DoH, and $r_{E 1}$ and $r_{E 3}$ represent radius ranges of the target blob for which DoH Filter is required to be searched.

\subsection{Comparative Experiment of Image Set A}

Image Set $\mathrm{A}$ has a total of 10 images named $A_{1}, A_{2}, \ldots$, $A_{10} . A_{1}$ denotes the image of noiseless standard binary blob (the 1st Row, 1st Column in Fig. 4), and the remaining 9 pieces are images acquired by applying the salt-and-pepper noise and Gaussian noise to Image $A_{1}$ respectively. Image $A_{1}$ contains a total of 33 types of binary blob shapes including 11 shapes and 3 sizes, and the blobs of each size have circumscribed circles with the same radius. The rightmost column of Image $A_{1}$ contains two small-sized discs, which are connected by a thin line that may be considered as the noise. The radius of the blob between the first and second lines in Image $A_{1}$ is 17 pix, the radius of the blob between the 3rd and 4th lines is 26 pix, and the radius of the blob between the 5th and 6th lines is 38 pix. $A_{2} \sim A_{4}$ are images after being applied 5\%,10\%, and 15\% salt-and-pepper noise respectively. $A_{5} \sim A_{7}$ are images acquired by applying Gaussian noise with variances in 10,30 and 50 respectively. $A_{8} \sim A_{10}$ are images acquired by applying $5 \%$ saltand-pepper noise + Gaussian noise with the variance of 10 , $10 \%$ of salt-and-pepper noise + Gaussian noise with the variance in 30 , and $15 \%$ of salt-and-pepper noise + Gaussian noise with the variance in 50. All Gaussian noises have the mean value in 0 and the intensity in 150 . The three images in the first column of Fig. 4 are $A_{1}, A_{9}$ and $A_{10}$ respectively.

The purpose of this experiment is to compare the antinoise ability of SMBD and the other four blob detection algorithms by detecting blobs with the radius of approximately 26 pixels (the blobs in the third to fourth lines in the images in the first column of Fig. 4) in Image Set A. The second column of Fig. 4 is the result of blob detection of the image of the first column on the left side with SMBD. It can be concluded from the figure that all the target blobs (13 in total) are detected, and the shapes of detected blobs are extremely complete. In addition, other blobs with smaller radiuses are also detected. The parameters of the SMBD in the experiments are $r_{A 1}=17, r_{A 4}=26, r_{A 2}=r_{A 4}+2$, $r_{A 3}=r_{A 2}+1, T h_{A 1}=60, T h_{A 2}=10$, and the remaining parameters $k_{a}, k_{b}$, and $k_{d}$ take different values depending on the noise situation.

The third column of Fig. 4 represents the results of blob detection on images of the first column on the left side by using Quoit, of which the parameter values of the filter are the same as SMBD. As shown in the figure, the remaining noise images fail to detect the target blob except for the 11 target blobs detected in Image A1, which indicates that the Quoit is extremely sensitive to noise.

The image in the fourth column of Fig. 4 is the result of blob detection on images of the first column by the NQuoit. The filter parameters are: $r_{C 1}=26+2, r_{C 2}=r_{C 1}+1$, $T h_{C 1}=60$, and such parameters are similar corresponding parameters of SMBD. It can be concluded from the figure that a considerable number of target blobs are not detected, indicating that N-Quoit is also extremely sensitive to the noise.

Columns 5-6 of Fig. 4 represent the results of blob detection on images of the first column on the left side by LoG and DoH respectively. The filtering parameters satisfy $r_{D 1}=r_{E 1}=17, r_{D 2}=r_{E 2}=26, r_{D 3}=r_{E 3}=38$. There are many candidates for blob location points (more than 800 blobs per image on average), and only the point candidates 

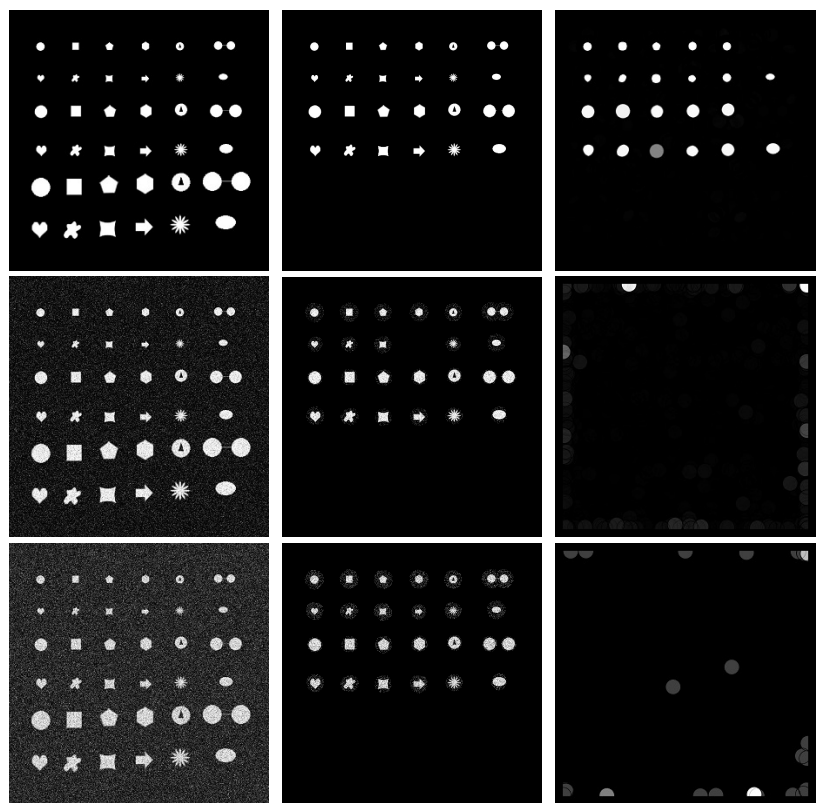
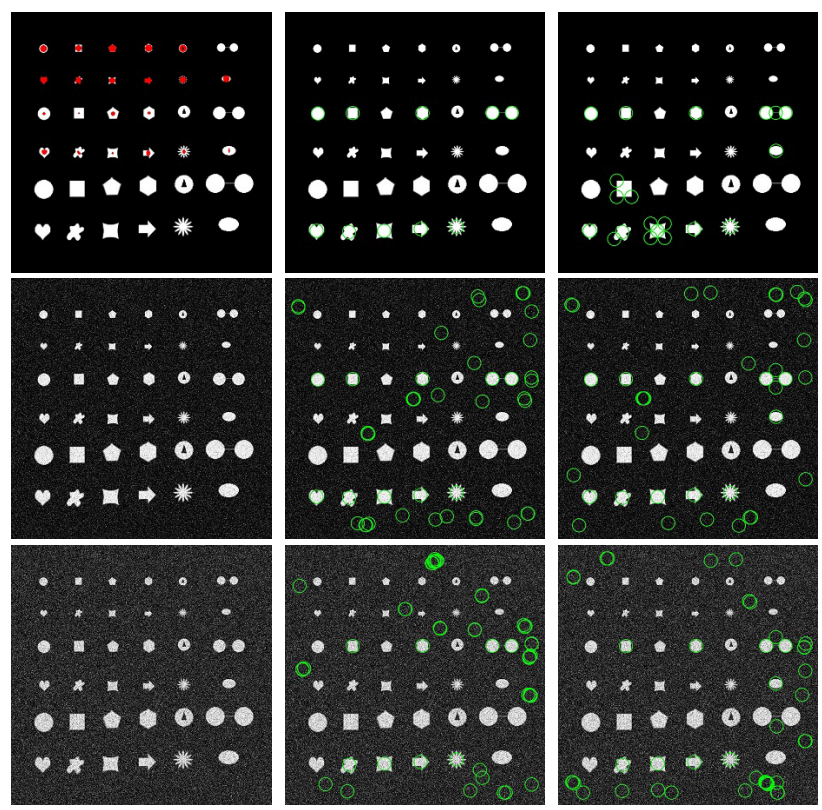

Fig. 4 Some experimental results of image set A. The first column involves three images of image set $\mathrm{A}$, and the columns from the second column to the sixth column are the results of blob detection on the images in the left column with SMBD, Quoit, N-Quoit, LoG, and DoH respectively.

Table 2 Detection rate of five detection algorithms for blob

\begin{tabular}{c|c|c}
\hline Name of algorithm & $\begin{array}{l}\text { Accuracy of Image Set A } \\
\text { total number of blobs is 130) }\end{array}$ & $\begin{array}{l}\text { Accuracy of Image Set B } \\
\text { total number of blobs is 246) }\end{array}$ \\
\hline SMBD & $100 \%$ & $98.78 \%$ \\
\hline Quoit & $33.84 \%$ & $86.58 \%$ \\
\hline N-Quoit & $30.77 \%$ & $88.62 \%$ \\
\hline LoG & $36.92 \%$ & $63.01 \%$ \\
\hline DoH & $42.31 \%$ & $59.35 \%$ \\
\hline
\end{tabular}

within the first 100 blobs are taken in the experiment for this reason, the green circle is represented on original drawing with the radius in 26 pix and the candidate location point as the circle center. It can be seen from the figure that considerable parts of target blobs are not detected.

There are a total of 130 target blobs with the radius in 26 pix on Image Set A. The data of comparison experiments of five blob detection algorithms are shown in Table 2. The performance of SMBD Algorithm is better than those of other four algorithms: SMBD is capable of fully detecting all target blobs from different noise images, and the detected blob shape is quite complete. The success of the blob detection is determined by visual inspection as follows: For SMBD and Quoit, the shapes of detected blobs are mostly close to original shapes, and most overlapping of original blob region is defined as successfully detected; For N-Quoit, LoG, and DoH, the distance between detected blob positon points of blob and the center of target blob less than $40 \%$ of the radius of target blob shall be defined as success.

Figure 4 shows the results of blob detection on Image Set A with five kinds of blob detecting algorithm: SMBD, Quoit, N-Quoit, LoG and HoG (part of the images). SMBD and Quoit output the blob shape to be detected, while NQuoit, LoG and HoG output the anchor point of the blob area to be detected. Therefore, in Fig. 4, column 2-3 list the blob shape detected by SMBD and Quoit, while column 4-6 list the anchor point of blob detected by N-Quoit, LoG and Hog. N-Quoit can detect many anchor points for a certain blob area; for the sake of convenient observation, we have made the anchor point display on the original figure directly. LoG and HoG can only detect one anchor point for some blob sometimes, if we display the anchor points directly, they might be not very clear; therefore, we set the anchor point as the circle center and draw a circle on the original figure with the radius (of target blob) of 26. Following the same principle, we processed Fig. 6 also in the same way.

\subsection{Comparative Experiment of Image Set B}

In order to verify the performance of the SMBD algorithm in actual grayscale image, we created Image Set B (Fig. 5). The blob inside the red circle is the target blob expected to be detected, and the sizes of target blobs in the same image are basically equivalent. Image Set B is produced as follows: Select 46 images from the disclosed saliency region test image library, and then cut out the blob subimages having different sizes and shapes and convert them into grayscale images.

Image Set B includes the small targets (blobs) of various shape. In the experiment, we randomly selected the small targets of different shapes and sizes (such as horse, window and special texture patterns) as the target blobs, with the purpose of examining the performance of blob detecting of SMBD using blobs of different shapes and sizes. Besides, the same image might include several kinds of blobs with different shapes and sizes, and we also randomly 


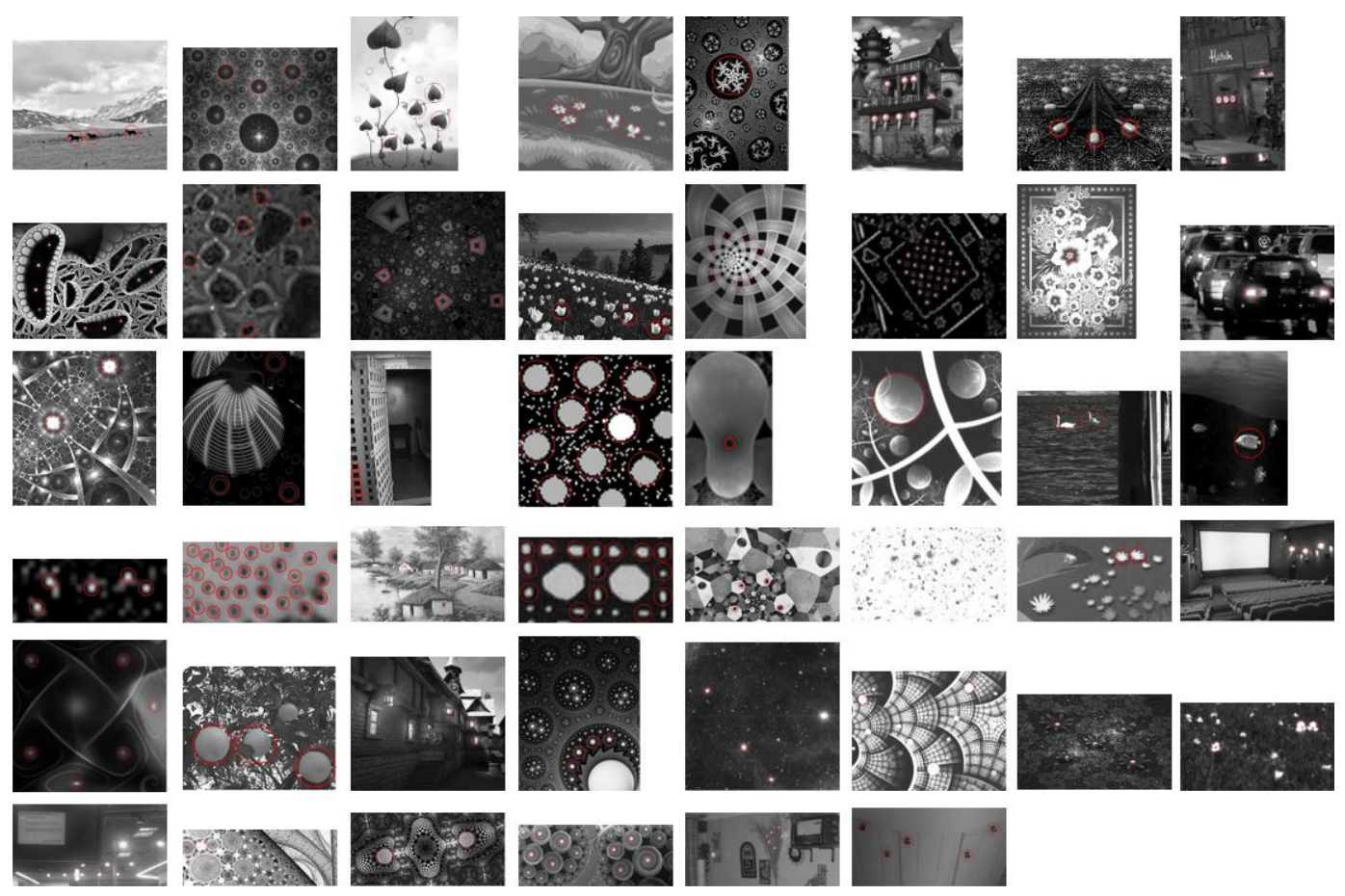

Fig. 5 All images of image set B. The blob inside the red circle is the target blob expected to be detected, and the sizes of target blobs in the same image are basically equivalent.
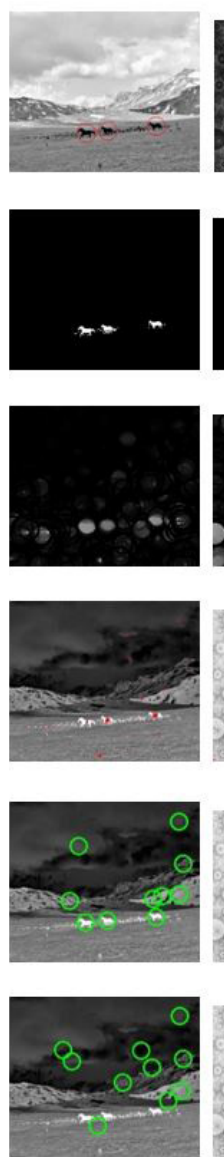
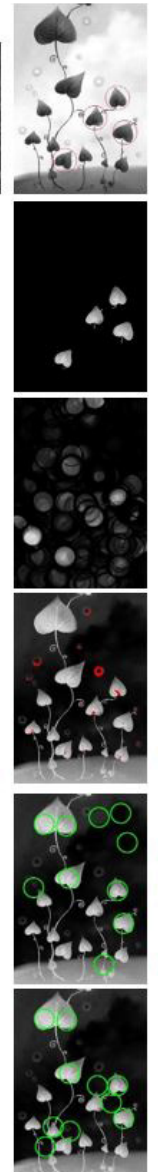
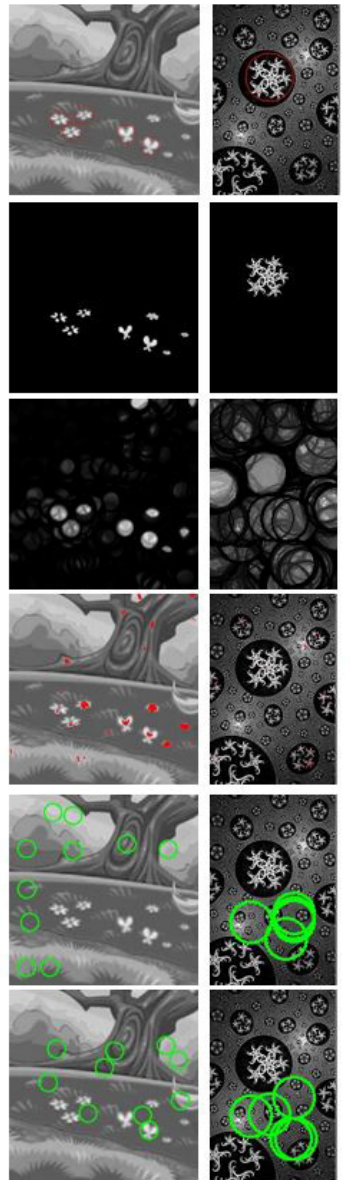
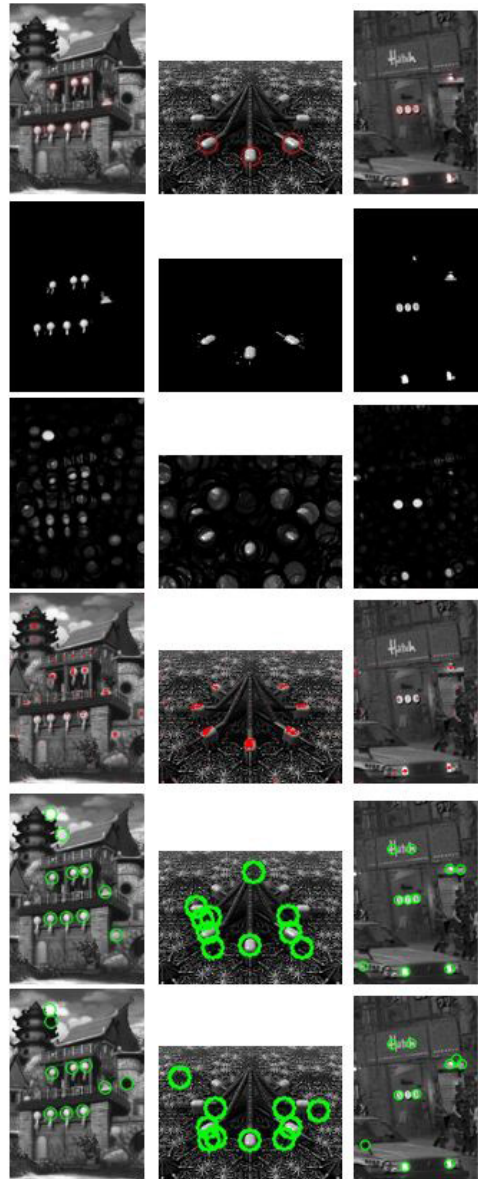

Fig. 6 Some experimental results of image set B. The first row involves eight images in image set B, and the second to the sixth rows are results of blob detection on images in the first row with SMBD, Quoit, N-Quoit, LoG and DoH algorithms respectively. 
selected some kind of small targets with basically similar shapes and sizes as the target blob (the area surrounded by red circle in Fig. 5). Such deal is fair for various kinds of blob detecting algorithm, and it means that the more blobs the algorithm can detect from Image Set B, the better performance it will have.

The purpose of this experiment is to compare the performance of SMBD to that of other four blob detection algorithms by detecting actual blobs (in different backgrounds, shapes, sizes, and grayscale changes) in Image Set B. In order to guarantee that experimental results of detection algorithms are comparable to utmost degree, the parameters are set as follows for all the images detected. The parameters of SMBD: $r_{A 2}=r_{T}+2, r_{A 3}=r_{A 2}+1, r_{A 4}=r_{T}, T h_{A 1}$ and $T h_{A 2}$ are set according to actual image conditions, $r_{A 1}$ is much less than $r_{T}$, and $r_{T}$ represents the radius of target blob. The parameters of Quoit: $r_{B 1}=r_{T}, r_{B 2}=r_{B 1}+2$, $r_{B 3}=r_{B 2}+1, T h_{B 1}=T h_{A 1}$. The parameters of N-Quoit: $r_{C 1}=r_{T}+2, r_{C 2}=r_{C 1}+1, T h_{C 1}=T h_{A 1}$. The parameters of LoG and DoH: $r_{D 2}=r_{E 2}=r_{T}, r_{D 1}=r_{E 1}=r_{T}-10(\geq 1)$, $r_{D 3}=r_{E 3}=r_{T}+10$.

The first row of Fig. 6 involves eight images in Image Set B, and the second to sixth rows of Fig. 6 are results of blob detection on images of first line of Fig. 6 with SMBD, Quoit, N-Quoit, LoG and DoH respectively. As there are numerous point candidates for blob detected by $\mathrm{LoG}$ and $\mathrm{DoH}$, only the first 100 candidates for location points of blobs are selected in the experiment, and they are represented in original images by use of green circles.

There are a total of 246 target blobs in Image Set B. The data of comparison experiment on five blob detection algorithms are shown in Table 2. The performance of SMBD algorithm is significantly better than that of other four algorithms. Correct detection rate of SMBD hits $98.78 \%$, and the shapes of blobs detected are extremely complete. In addition, the number of blob candidates detected with SMBD Algorithm is extremely close to the number of target blobs, while the number of blob candidates detected by the other four algorithms is significantly higher than that of SMBD. Whether the blobs in Image Set B have been successfully detected is determined by visual inspection and the criteria is as the same of that of Image Set A.

\subsection{The Operating Time of SMBD}

We have performed 50 repetitive experiments with 5 kinds of blob detecting algorithms to ten images $(1024 \times 1024$ gray scale image) in Image Set A. The parameters of the experiments of 5 kinds of blob detecting are the same as those of the comparison experiment in 4.1 , and the target blobs to be detected are also the same. Based on the experiments, we have obtained the average detectng time of these 5 kinds of blob detector, as is shown in the Table 3. The program

Table 3 Comparison of detection time \begin{tabular}{c|c|c|c|c|c} 
Name of algorithm & SMBD & Quoit & N-Quoit & LoG & DoH \\
\hline Average detection time (s) & 12.98 & 12.07 & 0.839 & 24.97 & 59.33
\end{tabular}

is realized with $\mathrm{C}++$, and the operation environment for the experiment is: i5-4590CPU, RAM 8GB, Windows 10 Professional. From the Table 3 we can see that, the detecting time of SMBD is similar with that of Quoit, and it is slower than N-Quoit, however, it is better than LOG and DoH.

\section{Conclusions}

The dilation operators of standard mathematical morphology are extended to the order dilation operator, and three soft morphological filters are designed, based on which SMBD Algorithm is proposed. Though SMBD, Quoit and $\mathrm{N}$-Quoit are algorithms with the same type based on mathematical morphology, theoretically, SMBD is featured with greater anti-noise capacity than that of Quoit and N-Quoit. According to the results of comparison experiment, SMBD not only features with extremely powerful anti-noise capacity but also quite complete blob shape detected compared to other four algorithms, and the number of blob candidates are extremely close to original number of blobs. Theoretically, the blob with the radius within specified range may be detected by two times of SMBD filtering if the target blob is a connected element region. SMBD has 12 parameters, among which 4 parameters of structural element may be easily set according to the size of target blob, whereas the remaining 8 parameters are required to be set according to the image conditions. It is a topic to be explored in the future to realize the adaptive processing of such parameters.

\section{References}

[1] S. Yamamoto, M. Matsumoto, Y. Tateno, T. Iinuma, and T. Matsumoto, "Quoit filter-a new filter based on mathematical morphology to extract the isolated shadow, and its application to automatic detection of lung cancer in x-ray ct," Proc. 13th International Conference on Pattern Recognition 1996, vol.2, pp.3-7, 1996.

[2] T. Miwa, J.-I. Kako, S. Yamamoto, M. Matsumoto, Y. Tateno, T. Iinuma, and T. Matsumoto, "Automatic detection of lung cancers in chest ct images by the variable n-quoit filter," Systems and Computers in Japan, vol.33, no.1, pp.53-63, 2002.

[3] T. Kurzendorfer, P.W. Mewes, A. Maier, N. Strobel, and A. Brost, "Cryo-balloon catheter localization based on a support-vector-machine approach," IEEE Trans. Med. Imag., vol.35, no.8, pp.1892-1902, 2016.

[4] M. Zhang, T. Wu, S.C. Beeman, L. Cullen-McEwen, J.F. Bertram, J.R. Charlton, E. Baldelomar, and K.M. Bennett, "Efficient small blob detection based on local convexity, intensity and shape information,” IEEE Trans. Med. Imag., vol.35, no.4, pp.1127-1137, 2016.

[5] G. Zhang, J. Liu, Y. Liu, J. Zhao, L. Tian, and Y.Q. Chen, "Physical blob detector and multi-channel color shape descriptor for human detection," Journal of Visual Communication and Image Representation, vol.52, pp.13-23, 2018.

[6] P. Afsar, P. Cortez, and H. Santos, "Automatic human trajectory destination prediction from video," Expert Systems with Applications, vol.110, pp.41-51, 2018.

[7] Y. Hu, Z. Long, and G. AlRegib, "A high-speed, real-time vision system for texture tracking and thread counting," IEEE Signal Process. Lett., vol.25, no.6, pp.758-762, 2018.

[8] A. Durand-Petiteville, S. Vougioukas, and D.C. Slaughter, "Realtime segmentation of strawberry flesh and calyx from images of singulated strawberries during postharvest processing," Computers and Electronics in Agriculture, vol.142, pp.298-313, 2017. 
[9] K. Rajasekaran, E. Samani, M. Bollavaram, J. Stewart, and A.G. Banerjee, "An accurate perception method for low contrast bright field microscopy in heterogeneous microenvironments," Applied Sciences, vol.7, no.12, p.1327, 2017.

[10] V. Ewald, R.M. Groves, and R. Benedictus, "Transducer placement option of lamb wave shm system for hotspot damage monitoring," Aerospace, vol.5, no.2, p.39, 2018.

[11] L. Xin, D. Luo, and H. Li, "A monocular visual measurement system for uav probe-and-drogue autonomous aerial refueling," International Journal of Intelligent Computing and Cybernetics, vol.11, no.2, pp.166-180, 2018.

[12] Y. Du, Y. Jiang, and W. Zhou, "An accurate two-step isar cross-range scaling method for earth-orbit target," IEEE Geosci. Remote Sens. Lett., vol.14, no.11, pp.1893-1897, 2017.

[13] T. Lindeberg, "Feature detection with automatic scale selection," International journal of computer vision, vol.30, no.2, pp.79-116, 1998.

[14] G.J. Hay, P. Dubé, A. Bouchard, and D.J. Marceau, "A scale-space primer for exploring and quantifying complex landscapes," Ecological Modelling, vol.153, no.1-2, pp.27-49, 2002.

[15] H. Kong, H.C. Akakin, and S.E. Sarma, "A generalized laplacian of gaussian filter for blob detection and its applications," IEEE Trans. Cybern., vol.43, no.6, pp.1719-1733, 2013.

[16] Z. Miao and X. Jiang, "Interest point detection using rank order log filter," Pattern Recognition, vol.46, no.11, pp.2890-2901, 2013.

[17] M. Zhang, T. Wu, and K.M. Bennett, "Small blob identification in medical images using regional features from optimum scale," IEEE Trans. Biomed. Eng., vol.62, no.4, pp.1051-1062, 2015.

[18] J. Matas, O. Chum, M. Urban, and T. Pajdla, "Robust wide-baseline stereo from maximally stable extremal regions," Image and vision computing, vol.22, no.10, pp.761-767, 2004.

[19] M.A. Duval-Poo, N. Noceti, F. Odone, and E. De Vito, "Scale invariant and noise robust interest points with shearlets," IEEE Trans. Image Process., vol.26, no.6, pp.2853-2867, 2017.

[20] G. Takacs, V. Chandrasekhar, S.S. Tsai, D. Chen, R. Grzeszczuk, and B. Girod, "Fast computation of rotation-invariant image features by an approximate radial gradient transform," IEEE Trans. Image Process., vol.22, no.8, pp.2970-2982, 2013.

[21] K.T.M. Han and B. Uyyanonvara, "A Survey of Blob Detection Algorithms for Biomedical Images," IEEE 2016 7th International Conference of Information and Communication Technology for Embedded Systems, 2016.

[22] L. Koskinen, J.T. Astola, and Y.A. Neuvo, "Soft morphological filters," Image Algebra and Morphological Image Processing II, vol.1568, pp.262-271, 1991.

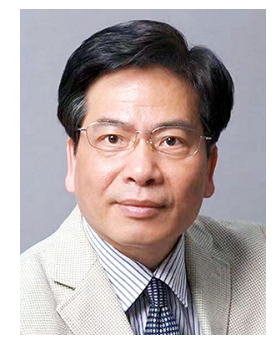

Weiqing Tong received the B.E. degree in Control Science and Engineering from Shanghai University, China, in 1983, and the M.S. and the Ph.D. degree in Computer Science and Technology from Osaka City University, Japan, in 1995 and 1998 respectively. He worked at Sinopec Shanghai Petrochemical Company Limited, China, from 1983 to 1990 , and worked as a researcher at NEC Informatec Systems, Ltd., Japan, from 1998 to 2004. He is currently an associate professor at Department of Computer science and technology, East China Normal University, China. His main research interests include computer vision, image processing and machine learning.

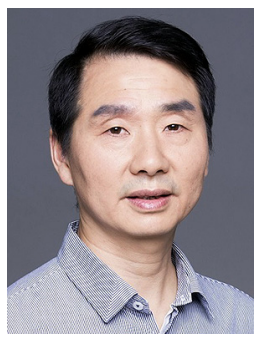

Haisheng Li received B.S., M.S. and Ph.D. degrees in Department of Mathematics from Zhejiang University in 1990, 1993 and 2003 respectively. Presently, he is an associate professor at Department of Computer science and technology, East China Normal University, China. His research interests include computer aided filament winding, computer graphics, and image processing.

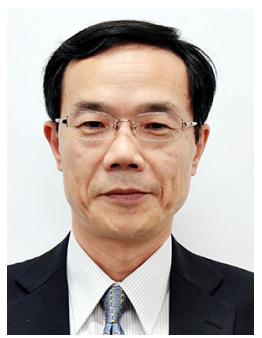

Guoyue Chen received his B.S. degree from East China Normal University, China, in 1983, where he worked as a research associate at the Department of Computer Science until 1989. He received his M.S. and Ph.D degrees from Tohoku University, Japan in 1993 and 1996, respectively. He is currently a professor in Akita Prefecture University, Japan. His interests include digital signal processing and its applications to active noise control, and image processing. 